\title{
Effect of artificial saliva contamination on adhesion of dental restorative materials
}

\author{
Kisaki SHIMAZU1', Hiroyuki KARIBE ${ }^{1}$ and Kiyokazu OGATA ${ }^{1,2}$ \\ ${ }^{1}$ Department of Pediatric Dentistry, The Nippon Dental University School of Life Dentistry at Tokyo, 1-9-20 Fujimi, Chiyoda-ku, Tokyo 102-8159, \\ Japan \\ ${ }^{2}$ Division of Dentistry, Tokyo Metropolitan Children's Medical Center, 2-8-29 Musashidai, Fuchu-shi, Tokyo 183-8561, Japan \\ Corresponding author, Kisaki SHIMAZU; E-mail: kisaki-s@tky.ndu.ac.jp
}

\begin{abstract}
The purpose of this study was to evaluate the effects of artificial saliva contamination on three restorative materials, namely, a glass ionomer cement (GIC), a resin-modified GIC (RMGIC), and a composite resin (CR), for which two different etching adhesive systems were used. Thus, three surface conditions were created on bovine teeth using artificial saliva: control, mild saliva contamination, and severe saliva contamination. The dentin bond strength for CR was significantly lower after artificial saliva contamination. There were, however, no significant differences among the three surface conditions in terms of the dentin and enamel bond strengths of GIC and RMGIC. Moreover, CR exhibited significantly greater microleakage after artificial saliva contamination, whereas no significant differences were found in GIC and RMGIC. The results showed that artificial saliva contamination did not affect the shear bond strengths of GIC and RMGIC or their degrees of microleakage.
\end{abstract}

Keywords: Saliva contamination, Glass ionomer cements, Composite resins, Bond strength, Microleakage

\section{INTRODUCTION}

Contamination of the dental operation area by saliva and other fluids is detrimental to positive restoration and should be controlled. The use of a rubber dam enables the dentist to control the contamination and focus on the actual clinical procedure ${ }^{1-3)}$. However, it can be difficult to place a rubber dam on a severely fractured tooth, an erupting tooth, or the tooth of a child who is respiring orally. In addition, the rubber dam clamp may be uncomfortable for the patient ${ }^{4,5}$. Many dentists, therefore, refrain from using rubber dams during restorative care ${ }^{1)}$, which significantly increases the possibility of saliva contamination.

Recent developments in the field of dental materials have created a shift in restorative practices, which are now evidence-based and follow minimal-intervention policies ${ }^{6,7)}$. Composite resins (CRs) are commonly used, even though their bond strength is reduced when contaminated by saliva either before or after primer application $^{8)}$. On the other hand, glass ionomer cements (GICs) chemically adhere to mineralized dental tissues; however, incomplete chemical reactions and sensitivity to water during the first stage of the GIC-setting reaction can lead to the softening and cracking of the cement surface, which in turn, leads to a decrease in the wear resistance and fracture toughness of the $\mathrm{GICs}^{9}$. Resin-modified GICs (RMGICs) can be used to overcome these shortcomings. Yamazaki et al. have suggested that RMGICs have a greater shear bond strength than conventional GICs in the case of luting by water immersion ${ }^{10)}$.

One of the biggest problems with restoration materials is microleakage, which often results from marginal gaps between the restoration material and the cavity walls ${ }^{11)}$. These gaps are a result of inadequate wetting or spreading of the restoration material along the cavity walls during placement. Microleakage can also develop from temperature disparity if the dental structure and restoration material have different coefficients of thermal expansion ${ }^{12}$. The presence of gaps and the subsequent decrease in bond strength may result in the formation of secondary caries around the restorative material. Rekha et $a l .{ }^{13)}$ reported that RMGICs exhibit less microleakage than GICs after thermocycling. However, few studies have compared GICs, RMGICs, and CRs in terms of bond strengths and degrees of microleakage in a moist environment similar to that encountered during dental restoration.

The purpose of this in vitro study was to evaluate the effect of artificial saliva contamination on the shear bond strengths of three restorative materials - GIC, RMGIC, and CR - used for enamel or dentin restoration and the effect on the degree of microleakage exhibited by the restorative materials after thermocycling.

\section{MATERIALS AND METHODS}

The materials used in this study, GIC (Fuji IX extra capsule [F9E], GC Corp., Tokyo, Japan), RMGIC (Fuji II LC capsule [2LC], GC Corp.), and CR (CLEARFIL AP-X, Kuraray Noritake Dental Inc., Tokyo, Japan), are listed in Table 1. In the case of the CR, we used two different etching adhesive systems: a total-etching adhesive (OptiBond Solo Plus [CR-OBS], Kerr Corp., Orange, CA, USA) and a self-etching primer (CLEARFIL S3 BOND ND [CR-TSB], Kuraray Noritake Dental Inc.).

Various surface conditions were created on the enamel or dentin, or both, using artificial saliva. The artificial saliva contained $20 \mathrm{mM}$ 4-(2-hydroxyethyl)- 
Table 1 Materials used in the study

\begin{tabular}{|c|c|c|}
\hline Material & Composition (batch no.) & Application conditions \\
\hline $\begin{array}{l}\text { Fuji IX extra capsule } \\
\text { (F9E) }\end{array}$ & $\begin{array}{l}\text { Powder: fluoroaluminosilicate glass, } \\
\text { polycarboxylic acid. } \\
\text { Liquid: polycarboxylic acid, water, } \\
\text { polybasic carboxylic acid. } \\
\text { Shade: A3 (1202251). }\end{array}$ & - \\
\hline $\begin{array}{l}\text { Fuji II LC capsule } \\
(2 \mathrm{LC})\end{array}$ & $\begin{array}{l}\text { Powder: fluoroaluminosilicate glass. } \\
\text { Liquid: methacrylic acid ester, } \\
\text { polycarboxylic acid, water. } \\
\text { Shade: A3 (1210111). }\end{array}$ & - \\
\hline Cavity conditioner & $\begin{array}{l}\text { Water, polycarboxylic acid, aluminum chloride } \\
\text { (1212071). }\end{array}$ & $\begin{array}{l}\text { Apply the conditioner for } 10 \mathrm{~s} \text {, } \\
\text { rinse for } 10 \mathrm{~s} \text {, and gently air dry. }\end{array}$ \\
\hline CLEARFIL AP-X & $\begin{array}{l}\text { Monomer: Bis-GMA, TEGDMA. } \\
\text { Fillers: surface-treatment glass powder, } \\
\text { surface-treatment silica-based micro filler. } \\
\text { Additional contents: photo-initiator, colorant. } \\
\text { Shade: A3 (01432A). }\end{array}$ & - \\
\hline $\begin{array}{l}\text { OptiBond Solo Plus } \\
\text { (CR-OBS) }\end{array}$ & $\begin{array}{l}\text { Bond: Bis-GMA, hydroxyethyl methacrylate, } \\
\text { ethyl alcohol, fillers (4716918). } \\
\text { Gel etchant: } 37.5 \% \text { phosphoric } \\
\text { acid (4693004). }\end{array}$ & $\begin{array}{l}\text { Apply the etchant for } 15 \mathrm{~s} \text {, } \\
\text { rinse for } 15 \mathrm{~s} \text {, and gently air dry. } \\
\text { Bond for } 15 \mathrm{~s} \text {, gently air dry for } 10 \mathrm{~s} \text {, } \\
\text { and cure under light for } 20 \mathrm{~s} \text {. }\end{array}$ \\
\hline $\begin{array}{l}\text { CLEARFIL S3 BOND ND } \\
\text { (CR-TSB) }\end{array}$ & $\begin{array}{l}\text { Monomer: Bis-GMA, MDP, HEMA. } \\
\text { Filler: silica-based microfiller. } \\
\text { Additional contents: ethyl alcohol, photo-initiator, } \\
\text { water (00040C). }\end{array}$ & $\begin{array}{l}\text { Apply the primer and leave it in } \\
\text { place for } 20 \mathrm{~s} \text {, dry by blowing } \\
\text { high-pressure air for } 5 \mathrm{~s}, \\
\text { and cure under light for } 10 \mathrm{~s} \text {. }\end{array}$ \\
\hline
\end{tabular}

Abbreviations: Bis-GMA: bisphenol A-diglycidyl methacrylate; TEGDMA: triethylene glycol dimethacrylate; MDP: methacryloyloxydecyl dihydrogen phosphate; HEMA: 2-hydroxyethyl methacrylate; DMA: dimethacrylate.

1-piperazineethanesulfonic acid, $30 \mathrm{mM} \mathrm{KCl,} 4 \mathrm{mM}$ $\mathrm{KH}_{2} \mathrm{PO}_{4}$, and $0.7 \mathrm{mM} \mathrm{CaCl}_{2}{ }^{14)}$. The rationale for using artificial saliva was that the presence of calcium and phosphate ions would prevent additional demineralization, which can alter the etching depth for enamel and dentin surfaces treated with acid or adhesives. The artificial saliva did not contain sodium azide because there was no need to store the specimens in sodium azide. The test samples were divided into the following groups: Group I (control), where the bonding surface remained dry; Group II (mild saliva contamination), where $0.1 \mathrm{~mL}$ of the artificial saliva was placed on the bonding surface and dried slightly; and Group III (severe saliva contamination), where $0.1 \mathrm{~mL}$ of the artificial saliva was used as is.

\section{Shear bond strength test}

Bovine incisors were used in the study. After we had confirmed the absence of abnormalities such as discoloration or hypoplasia on their labial side, the bovine incisors were cut into blocks (cubes with sides of $5 \mathrm{~mm}$ ) using a low-speed diamond blade (IsoMet, Buehler Ltd, Lake Bluff, IL, USA). These blocks were embedded in an acrylic resin (Unifast II, GC Corp.). Next, the enamel or dentin surfaces of the blocks were ground with 120- and 320-grit sandpaper. The blocks were then divided into three groups: Group I, Group II, and Group III. Each of the three groups was then divided into four material categories: F9E, 2LC, CR-OBS, and CR-TSB. The surfaces of the F9E, 2LC, and CR-OBS blocks were treated in accordance with the instructions given in their respective manuals; a cavity conditioner was used for F9E and 2LC, and a 37.5\% phosphoric acid etch was used for CR-OBS. Artificial saliva was applied to the Group II and Group III blocks, and then the appropriate adhesives were applied to the CR-OBS and CR-TSB blocks, in accordance with their respective instructions. Ten specimens were prepared for each of the four materials in each of the three surface condition groups (i.e., a total of 120 specimens for each enamel and dentin). The specimens were prepared using a silicone ring-mold with an internal diameter of $2.4 \mathrm{~mm}$ and a height of $4.0 \mathrm{~mm}$. The 2LC, CR-OBS, and CR-TSB blocks were light cured for $20 \mathrm{~s}$ using a visible light-curing unit (G-Light Prima-II, GC Corp.), whereas the F9E blocks were stored for $5 \mathrm{~min}$ at $37^{\circ} \mathrm{C}$ and $100 \%$ relative humidity. After the silicone ring-molds were removed, all specimens were stored in water at $37^{\circ} \mathrm{C}$ for $24 \mathrm{~h}$.

The enamel and dentin shear bond strengths of the materials were tested using a universal testing machine 
(Autograph EZ-S, Shimadzu Corp., Kyoto, Japan) at a crosshead speed of $1.0 \mathrm{~mm} / \mathrm{min}$.

\section{Microleakage analysis}

Cavities (1.5 $\mathrm{mm}$ in depth and $3.0 \mathrm{~mm}$ in diameter) were created at the center of the labial aspect of the bovine incisor enamel. The specimens were randomly divided into one of the three surface condition groups, namely, Group I, Group II, and Group III. After the application of the artificial saliva, the cavities were filled with the respective materials (F9E, 2LC, CR-OBS, and CR-TSB) as described above $(\mathrm{N}=10)$. The filled cavities were covered with a polyester film. The $2 \mathrm{LC}$, CR-OBS, and CR-TSB blocks were irradiated for $20 \mathrm{~s}$, whereas the F9E blocks were stored for $5 \mathrm{~min}$ at $37^{\circ} \mathrm{C}$ and $100 \%$ relative humidity. Next, all of the specimens were stored for $24 \mathrm{~h}$ at $37^{\circ} \mathrm{C}$ in distilled water. The polyester film was then removed, and the specimens were gently polished using \#600 silicon carbide paper under water irrigation. All the specimens were thermocycled for 2000 cycles at $5^{\circ} \mathrm{C}$ and $55^{\circ} \mathrm{C}$; the dwell time was $30 \mathrm{~s}^{15,16)}$. Then, they were soaked in a $0.1 \%$ methylene blue solution at $37^{\circ} \mathrm{C}$ for $20 \mathrm{~h}$. The specimens were subsequently rinsed in distilled water, embedded in acrylic resin (Unifast II, GC Corp.), sectioned longitudinally on either side of the cavity midline using a low-speed diamond blade (IsoMet, Buehler Ltd.), and examined under 60× magnification using a digital microscope (VHX-100, Keyence Corp., Osaka, Japan). The degree of dye penetration in all four surfaces was scored on a scale of 0 to 3 in accordance with the ISO/TS 11405:2003 standard, which has the following definitions: 0 , no dye penetration; 1 , dye penetration into the enamel part of the cavity wall; 2 , dye penetration into the dentinal part of the cavity wall but not into the cavity floor; 3 , dye penetration into the cavity floor ${ }^{17)}$.

\section{Statistical analysis}

The enamel and dentin shear bond strengths corresponding to the three surface conditions for each material were analyzed using one-way analysis of variance (ANOVA). To determine the independent and combined effects of surface condition and material, the groups were compared via two-way ANOVA. In the cases where the ANOVA results indicated a significant difference, Tukey's honestly significant difference (HSD) adjustment for multiple comparisons was applied to determine the group differences. The $p$ values below 0.05 were considered significant.

The dye-penetration scores for the three surface conditions and for each of the four materials (F9E, 2LC, CR-OBS, and CR-TSB) were compared using the Kruskal-Wallis test. If a significant difference was found, the Mann-Whitney $U$-test was performed on the pairs of dye-penetration scores in each group. Because either three or six pair-wise planned comparisons were made, $p<0.016$ (i.e., $0.05 / 3$ ) and $p<0.008$ (i.e., 0.05/6) were considered significant values for the surface conditions and the materials, respectively. All of the analyses were performed using the statistical software package IBM SPSS Statistics ver. 21 (IBM Japan, Tokyo, Japan).

\section{RESULTS}

Figure 1 shows the enamel and dentin shear bond strength values of the tested materials for the different surface conditions. For CR-TSB, the enamel bond
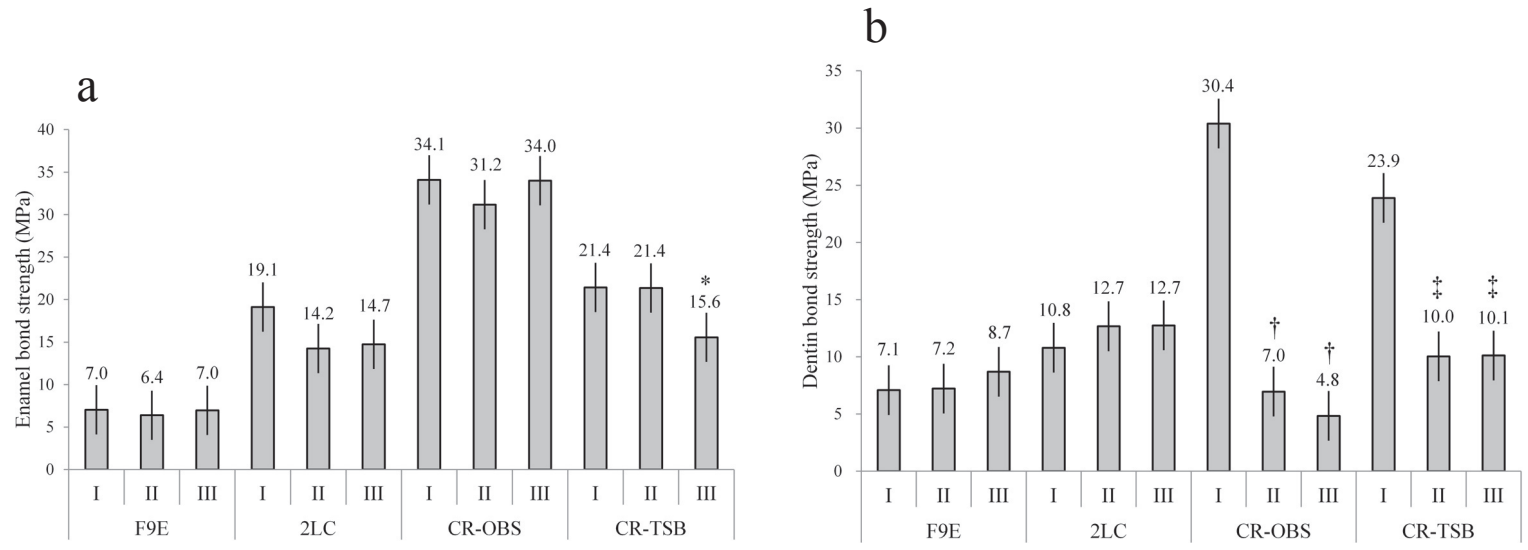

Fig. 1 (a) Enamel shear bond strengths and (b) dentin shear bond strengths of different materials under three surface conditions, Group I (the control), Group II (mild saliva contamination), and Group III (severe saliva contamination). The data shown represent the mean (SD) values for the following materials: F9E: Fuji IX extra capsule (glass ionomer cement); 2LC: Fuji II LC (resin-modified glass ionomer cement); CR-OBS: CLEARFIL AP-X (composite resin) using OptiBond Solo Plus (total-etching adhesive); and CR-TSB: CLEARFIL AP-X (composite resin) using CLEARFIL S3 BOND ND (self-etching primer).

* Significant differences in enamel bond strengths compared with Group I of CR-TSB $(p<0.01)$.

$\dagger$ Significant differences in dentin bond strengths compared with Group I of CR-OBS $(p<0.001)$.

¥ Significant differences in dentin bond strengths compared with Group I of CR-TSB $(p<0.001)$. 
strength was significantly lower in Group III than in Group I $(p<0.01)$, whereas there were no significant differences found for CR-OBS (Fig. 1a). Furthermore, for CR-OBS and CR-TSB, the dentin bond strengths were significantly lower in both Group II and Group III than in Group I (control) $(p<0.001$ for both materials) (Fig. 1b). On the other hand, F9E and 2LC exhibited no significant differences in either enamel or dentin bond strengths (Figs. 1a and 1b). The two-way ANOVA results indicated that the main effect of the surface condition was significant for enamel and dentine bond strength (enamel, $F(2,108)=3.111, p=0.049$; dentine, $F(2,108)=160.536, p<0.001)$ (Figs. $1 \mathrm{a}$ and $1 \mathrm{~b})$. The results for the main effect of the material were also significant for enamel and dentine bond strength (enamel, $F(3,108)=195.919, p<0.001$; dentine, $F(3$, $108)=46.002, p<0.001)$ (Figs. $1 \mathrm{a}$ and $1 \mathrm{~b})$. The interaction effect (surface condition $\times$ material) was not significant for enamel bond strength (Fig. 1a), although it yielded significant results for dentin bond strength ( $F(6$, $108)=82.217, p<0.001)$ (Fig. 1b).

The dye-penetration scores of the specimens for the four materials and the significance of the three different surface conditions for each material are presented in Table 2. A comparison of the dye-penetration scores for all of the conditions of each material revealed that CR-OBS and CR-TSB exhibited significantly higher dyepenetration scores after artificial saliva contamination ( $p=0.001$ for CR-OBS; $p=0.005$ for CR-TSB in the comparison of Group I and Group II; $p<0.001$ for both materials in the comparison of Group I and Group III) (see Table 2). However, no significant differences were found for F9E and 2LC.

On comparing the dye-penetration scores of the four different materials for each surface condition, the median dye-penetration scores were $0,1,1$, and 1 for F9E, 2LC, CR-OBS, and CR-TSB, respectively, in Group I. In addition, F9E exhibited significantly less microleakage than 2LC, CR-OBS, and CR-TSB ( $p=0.001$, $p=0.002$, and $p=0.0001$, respectively). In Group II, the median dye-penetration scores were $0,1,1$, and 1 for F9E, 2LC, CR-OBS, and CR-TSB, respectively. F9E exhibited significantly less microleakage than CROBS and CR-TSB ( $p<0.001$ for both materials). 2LC exhibited significantly less microleakage than CR-TSB $(p=0.001)$. In Group III, the median dye-penetration scores were 1, 1, 2, and 2 for F9E, 2LC, CR-OBS, and CR-TSB, respectively. F9E exhibited significantly less microleakage than CR-OBS and CR-TSB $(p<0.001$ for both materials). 2LC exhibited significantly less microleakage than CR-OBS and CR-TSB $(p<0.001$ for both materials).

\section{DISCUSSION}

In the present study, we compared the shear bond strengths and degrees of microleakage in three restorative materials (GIC, RMGIC, and CR) under artificial saliva contamination. The main finding of this study is that artificial saliva contamination did not affect

Table 2 Microleakage scores for different materials under three surface conditions

\begin{tabular}{|c|c|c|c|c|c|c|c|}
\hline \multirow{2}{*}{ Material * } & \multirow{2}{*}{ Group ** } & \multicolumn{4}{|c|}{ Score (total of 40 surfaces) ${ }^{\dagger}$} & \multirow{2}{*}{ Median } & \multirow{2}{*}{ Mean } \\
\hline & & 0 & 1 & 2 & 3 & & \\
\hline \multirow{3}{*}{ F9E } & I & 28 & 11 & 1 & 0 & 0 & 0.33 \\
\hline & II & 21 & 17 & 2 & 0 & 0 & 0.53 \\
\hline & III & 19 & 17 & 4 & 0 & 1 & 0.63 \\
\hline \multirow{3}{*}{$2 \mathrm{LC}$} & I & 15 & 17 & 8 & 0 & 1 & 0.83 \\
\hline & II & 12 & 23 & 5 & 0 & 1 & 0.83 \\
\hline & III & 10 & 25 & 5 & 0 & 1 & 0.88 \\
\hline \multirow{3}{*}{ CR-OBS } & I & 14 & 24 & 2 & 0 & 1 & 0.70 \\
\hline & II & 5 & 23 & 12 & 0 & 1 & $1.18^{\ddagger}$ \\
\hline & III & 5 & 12 & 20 & 3 & 2 & $1.53 \ddagger$ \\
\hline \multirow{3}{*}{ CR-TSB } & I & 4 & 33 & 3 & 0 & 1 & 0.98 \\
\hline & II & 3 & 22 & 13 & 2 & 1 & $1.35^{\S}$ \\
\hline & III & 0 & 17 & 20 & 3 & 2 & $1.65^{\S}$ \\
\hline
\end{tabular}

* F9E: Fuji IX extra capsule (glass ionomer cement); 2LC: Fuji II LC (resin-modified glass ionomer cement); CR-OBS: CLEARFIL AP-X (composite resin) using OptiBond Solo Plus (total-etching adhesive); CR-TSB: CLEARFIL AP-X (composite resin) using CLEARFIL S3 BOND ND (self-etching primer).

** Group I: control; Group II: mild saliva contamination; Group III: severe saliva contamination.

$\uparrow$ Score: 0, no dye penetration; 1 , dye penetration into the enamel part of the cavity wall; 2 , dye penetration into the dentine part of the cavity wall but not into the cavity floor; 3, dye penetration all the way into the cavity floor.

${ }^{\ddagger}$ Significant differences in dye-penetration scores from Group I of CR-OBS $(p<0.008)$.

$\S$ Significant differences in dye-penetration scores from Group I of CR-TSB $(p<0.008)$. 
the adhesion of GIC and RMGIC.

The results of statistical analyses showed that artificial saliva contamination decreased the dentin bond strengths of CR-OBS and CR-TSB; however, their enamel bond strengths were not significantly affected. The micromechanical bonds formed on the enamel surfaces that were demineralized by acid etching may explain the difference between the dentin and enamel bond strengths. However, the overall results prove the necessity of a rubber dam when using CR for the restorative treatment of a cavity that reaches into the dentin. On the other hand, artificial saliva contamination did not affect the dentin and enamel bond strengths for F9E and 2LC. Moreover, under artificial saliva contamination, the dentin bond strength was significantly higher in F9E than in CR-OBS and was significantly higher in 2LC than the other materials, although the enamel and dentin bond strengths of CROBS and CR-TSB were significantly higher than F9E and $2 \mathrm{LC}$ in the absence of artificial saliva contamination (control). These results may originate from the chemical self-adhesion of enamel and dentin owing to the presence saliva, even in the absence of applied adhesives. The adhesion mechanism involves the formation of ionic bonds between the glass ionomer and the calcium within the tooth structure ${ }^{18)}$. Conventional GICs undergo a chemical self-setting acid-base reaction when powdered fluoroaluminosilicate glass is mixed with aqueous polyacrylic acid or polycarboxylate acid. The chemistry of RMGICs is improved by the addition of methyl methacrylate or hydroxyethyl methacrylate, which are hydrophilic monomers, as the resin component. Compared to conventional GICs, RMGICs demonstrate improved adhesion to enamel and dentin, as well as significantly improved aesthetic properties. However, according to Cattani-Lorente et al. ${ }^{19)}$, the flexural strength and Vickers hardness of RMGICs are sensitive to their water content. Thus, further studies are necessary to investigate the flexural strength and hardness of restorative materials when contaminated by saliva.

Thermal changes naturally occur in vivo and can be simulated in the laboratory by thermocycling regimens, in which both the restoration and the tooth are subjected to extreme temperatures in vitro ${ }^{20,21}$. Microleakage may result from saliva contamination during setting, along with oral thermal changes after setting. The gaps that result may engender staining, marginal breakdown, hypersensitivity, secondary caries, and the development of pulpal pathology ${ }^{22}$. In the present study, the degree of microleakage for CR-OBS and CR-TSB after thermocycling increased significantly under artificial saliva contamination. The bonding materials (OBS and TSB) contain hydrophilic monomers, which enhance water sorption, and dying agents, the presence of which may explain the high degree of microleakage observed in this study. However, the degree of microleakage did not increase in F9E and 2LC and may not be affected by artificial saliva contamination because of the chemical self-adhesion in F9E and 2LC; bonding materials were not used. Moreover, F9E exhibited significantly less microleakage than the other materials in the absence of artificial saliva contamination (control). On the other hand, as a result of polymerization contraction and thermal changes, 2LC, CR-OBS, and CR-TSB exhibited microleakage under the control conditions.

The results of our study suggest that GIC and RMGIC are suitable for restorative treatment when isolation using a rubber dam is not feasible. Atraumatic restorative treatments (ARTs) are possible even when it is difficult to prevent contamination by saliva, such as in situations when a treatment is performed outside the dental clinic: only hand instruments are required, and the tooth can be restored with a $\mathrm{GIC}^{23}$. Alves et $a l .{ }^{23)}$ suggested that the presence of caries-affected dentin does not hinder the bonding of GICs to the primary tooth dentin.

\section{CONCLUSIONS}

On the basis of the results of the study, the following conclusions were drawn:

1. Contamination with artificial saliva does not affect the shear bond strengths of GICs and RMGICs; this is true for both bovine enamel and dentin.

2. The bond strength of CRs with bovine dentin is reduced significantly when a total-etching adhesive or a self-etching primer is used and the restorations are subjected to contamination with artificial saliva.

3. The microleakage in the case of cavities whose bovine enamel margin is filled with a GIC or an RMGIC does not increase under artificial saliva contamination after thermocycling.

\section{REFERENCES}

1) Ammann P, Kolb A, Lussi A, Seemann R. Influence of rubber dam on objective and subjective parameters of stress during dental treatment of children and adolescents — a randomized controlled clinical pilot study. Int J Paediatr Dent 2013; 23: 110-115.

2) Kameyama A, Asami M, Noro A, Abo H, Hirai Y, Tsunoda M. The effects of three dry-field techniques on intraoral temperature and relative humidity. J Am Dent Assoc 2011; 142: 274-280.

3) Slawinski D, Wilson S. Rubber dam use: a survey of pediatric dentistry training programs and private practitioners. Pediatr Dent 2010; 32: 64-68.

4) Iwatani K, Matsuo K, Kawase S, Wakimoto N, Taguchi A, Ogasawara T. Effects of open mouth and rubber dam on upper airway patency and breathing. Clin Oral Investig 2013; 17: 1295-1299.

5) Yoon RK, Chussid S. Topical anesthesia for rubber dam clamp placement in sealant placement: comparison of lidocaine/ prilocaine gel and benzocaine. Pediatr Dent 2009; 31: 377 381.

6) Frencken JE, Peters MC, Manton DJ, Leal SC, Gordan VV, Eden E. Minimal intervention dentistry for managing dental caries - a review: report of a FDI task group. Int Dent $\mathrm{J}$ 2012; 62: 223-243.

7) Momoi Y, Hayashi M, Fujitani M, Fukushima M, Imazato 
S, Kubo S, Nikaido T, Shimizu A, Unemori M, Yamaki C. Clinical guidelines for treating caries in adults following a minimal intervention policy-evidence and consensus based report. J Dent 2012; 40: 95-105.

8) Cobanoglu N, Unlu N, Ozer F, Blatz M. Bond strength of self-etch adhesives after saliva contamination at different application steps. Oper Dent 2013; 38: 505-511.

9) Molina GF, Cabral RJ, Mazzola I, Brain Lascano L, Frencken JE. Biaxial flexural strength of high-viscosity glass-ionomer cements heat-cured with an LED lamp during setting. BioMed Research International 2013; 2013: 838460.

10) Yamazaki A, Hibino Y, Honda M, Nagasawa $Y$, Hasegawa $Y$, Omatsu J, Yamaga T, Nakajima H. Effect of water on shear strength of glass ionomer cements for luting. Dent Mater J 2007; 26: 708-712.

11) Vicente A, Ortiz AJ, Parra PL, Calvo JL, Chiva F. Microleakage in Class $\mathrm{V}$ composite and compomer restorations following exposure to a colutory prescribed for the treatment of xerostomy. Odontology 2011; 99: 49-54.

12) Hakimeh S, Vaidyanathan J, Houpt ML, Vaidyanathan TK, Von Hagen S. Microleakage of compomer class V restorations: effect of load cycling, thermal cycling, and cavity shape differences. J Prosthet Dent 2000; 83: 194-203.

13) Rekha CV, Varma B, Jayanthi. Comparative evaluation of tensile bond strength and microleakage of conventional glass ionomer cement, resin modified glass ionomer cement and compomer: an in vitro study. Contemp Clin Dent 2012; 3: 282287.

14) Pashley DH, Tay FR, Yiu C, Hashimoto M, Breschi L, Carvalho RM, Ito S. Collagen degradation by host-derived enzymes during aging. J Dent Res 2004; 83: 216-221.

15) Eiriksson SO, Pereira PN, Swift EJ, Jr., Heymann HO,
Sigurdsson A. Effects of saliva contamination on resin-resin bond strength. Dent Mater 2004; 20: 37-44.

16) Ibarra G, Johnson GH, Geurtsen W, Vargas MA. Microleakage of porcelain veneer restorations bonded to enamel and dentin with a new self-adhesive resin-based dental cement. Dent Mater 2007; 23: 218-225.

17) ISO/TS 11405-2003: Dental materials - Testing of adhesion to tooth structure. International Organization for Standardization 2003.

18) Coutinho E, Yoshida $Y$, Inoue S, Fukuda R, Snauwaert J, Nakayama Y, De Munck J, Lambrechts P, Suzuki K, Van Meerbeek B. Gel phase formation at resin-modified glassionomer/tooth interfaces. J Dent Res 2007; 86: 656-661.

19) Cattani-Lorente MA, Dupuis V, Payan J, Moya F, Meyer JM. Effect of water on the physical properties of resin-modified glass ionomer cements. Dent Mater 1999; 15: 71-78.

20) Hitz T, Stawarczyk B, Fischer J, Hammerle CH, Sailer I. Are self-adhesive resin cements a valid alternative to conventional resin cements? A laboratory study of the longterm bond strength. Dent Mater 2012; 28: 1183-1190.

21) Schmid-Schwap M, Graf A, Preinerstorfer A, Watts DC, Piehslinger E, Schedle A. Microleakage after thermocycling of cemented crowns - a meta-analysis. Dent Mater 2011; 27: 855-869.

22) Mali P, Deshpande S, Singh A. Microleakage of restorative materials: an in vitro study. J Indian Soc Pedod Prev Dent 2006; 24: 15-18.

23) Alves FB, Hesse D, Lenzi TL, Guglielmi Cde A, Reis A, Loguercio AD, Carvalho TS, Raggio DP. The bonding of glass ionomer cements to caries-affected primary tooth dentin. Pediatr Dent 2013; 35: 320-324. 